\title{
Representations of the Heisenberg Algebra on a Riemann Surface $\star$
}

\author{
Arthur Jaffe, Slawomir Klimek, and Andrzej Lesniewski \\ Harvard University, Cambridge, MA 02138, USA
}

\begin{abstract}
We formulate reflection positivity for meromorphic functions and for 1-forms on a Riemann surface. This construction yields representations of the Heisenberg algebra on a Riemann surface.
\end{abstract}

\section{Introduction}

Conformal field theories on a Riemann surface with genus $\geqq 1$ have interesting features which are absent in the case of genus zero. One example of such a phenomenon is the nonuniqueness of basic representations of global chiral algebras. The simplest example of such an algebra is the Heisenberg algebra. Study of this algebra yields the theory of a free chiral field on a Riemann surface.

Krichever and Novikov understood the fact that the ordinary Heisenberg algebra, suitable for surfaces of genus zero, has to be extended [KN]. We study the resulting algebra in Sects. II and III; ultimately in Sect. V we describe global representations for such an algebra.

We restrict attention to Riemann surfaces $S$ which are Schottky doubles of an open Riemann surface $T$. On such a Riemann surface there is a natural notion of Osterwalder-Schrader positivity. In other words, there is an antiholomorphic involution $\vartheta$ which we can interpret as time reflection. We study a space $\mathfrak{U}$ of meromorphic functions on $S$. We use $\Theta$, the lift of $\vartheta$ to $\mathfrak{A}$, and a symplectic form $(\cdot, \cdot)$ on $\mathfrak{A}$; we then define an inner product $\langle\cdot, \cdot\rangle=(\Theta \cdot, \cdot)$ on a subalgebra $\mathfrak{U}_{+}$of $\mathfrak{U}$. The existence of an inner product allows us to construct representations of $\mathfrak{U}$ on a Fock-type Hilbert space. Our method uses certain ideas in [DVV].

Riemann surfaces which are Schottky doubles exist for any genus. In conformal field theory one has developed methods to compute arbitrary correlation functions once the operator formalism is known for certain simple surfaces. Here we do not use such sewing procedures, but rather give a direct, global construction.

\footnotetext{
* Supported in part by the Department of Energy under Grant DE-FG02-88ER25065
} 
Although our primary concern here is the Heisenberg algebra, one could investigate the representation theory for the integrated form of the algebra, namely for the global Weyl group, along the lines of [BKS].

\section{Reflection Positivity on a Schottky Double}

Let $S$ denote a compact Riemann surface which arises as a Schottky double of a bordered Riemann surface $T$ with boundary $\partial T$. Recall that the Schottky double $S$ of $T$ is defined in terms of its mirror image $\bar{T}$, and $S$ is the union of $\bar{T}$ with $T$ glued on $\partial T$. The Schottky double $S$ of $T$ has an antiholomorphic involution $\vartheta: S \rightarrow S$ such that $\vartheta T=\bar{T}$, and such that $\partial T$ is the set of fixed points of $\vartheta$. See [A] for details. Let $P_{0} \in T$ denote a point in general position and define $P_{\infty}=\vartheta\left(P_{0}\right)$. The points $P_{0}$ and $P_{\infty}$ provide reference points on the Riemann surface, which are interchanged by $\vartheta$, see Fig. 1.

The standard case of a real, two-dimensional, toroidal Euclidean space-time $S^{1}$ $\times \mathbb{R}$ can be understood as follows. For $t \in \mathbb{R}, x \in S^{1}$, let $(t, x)$ denote a space-time point. The map

$$
z=\exp [i(x+i t)]
$$

takes values in the Riemann sphere $S$, with the half-space $t \geqq 0$ mapping into the unit disc $T$ around the origin. Time reflection $(t, x) \rightarrow(-t, x)$ in the Euclidean spacetime maps into a reflection $\vartheta$ on the Riemann sphere. In local coordinates,

$$
\vartheta(z)=\frac{1}{\bar{z}} .
$$

We identify $\bar{T}$ as the unit disc about infinity. Note that the Riemann sphere is the Schottky double of the unit disc, with $\vartheta$ given by (II.2). A convenient choice for $P_{0}$ is $P_{0}=0$, so $P_{\infty}=\infty$, and this comes from Euclidean space-time points at $t=\mp \infty$. In particular, the operator $\vartheta$ on the Riemann sphere can be thought of as a reflection through the unit circle $|z|=1$. This toroidal space-time arises e.g. in the "radial quantization" of string theory.

The corresponding "infinite volume" space-time $(t, x) \in \mathbb{R}^{2}$ can also be studied. A compactification is given by the map

$$
z=\frac{x+i(t-1)}{x+i(t+1)}
$$

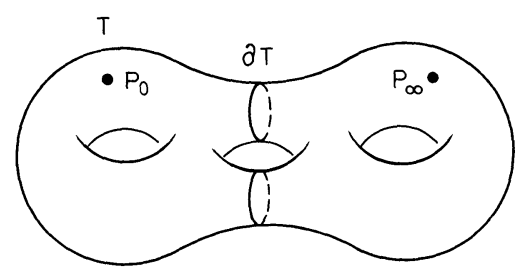

Fig. 1. The Schottky double $S$ of a bordered Riemann surface $T$ with boundary $\partial T$ 
Here the unit disc about the origin $T$ is the image of the positive time subspace while $\bar{T}$ is the image of the negative time subspace. The unit circle $\partial T$, which is the image of $t=0$, is invariant under $\vartheta$. The points $(t= \pm 1, x=0)$ are mapped into $P_{0}$, $P_{\infty}$.

On a general Schottky double $S$, the involution $\vartheta$ can also be interpreted as reflection in a unit circle. The notion of a radius (modulus) function for $S$ was introduced in [KN]. For every Riemann surface (not necessarily a Schottky double) there is a modulus function $R(z)$ taking values in $[0, \infty]$ such that $R\left(P_{0}\right)=0$ and $R\left(P_{\infty}\right)=\infty$. The existence of $R$ follows from the existence of a unique meromorphic form $\eta$ on $S$ with the following properties: (i) $\eta$ is holomorphic on $S \backslash\left\{P_{0}, P_{\infty}\right\} ; \operatorname{ord}_{P_{0}} \eta=\operatorname{ord}_{P_{\infty}} \eta=-1$, (ii) $\operatorname{Res}_{P_{0}} \eta=1$, (iii) $\operatorname{Re} \int_{C} \eta=0$, for every 1-cycle $C$, see Sect. II.5 of [FK]. For $P_{1} \in \partial T$, set [KL]

$$
R(z):=\exp \left(\operatorname{Re} \int_{P_{1}}^{z} \eta\right)
$$

Proposition II.1. With the above definitions,

$$
R(z) R(\vartheta(z))=1 .
$$

In particular $R(z)=1$, for $z \in \partial T$.

Proof. The meromorphic form $-\vartheta^{*} \bar{\eta}$ has the properties (i)-(iii). By uniqueness it must coincide with $\eta$. Since $\vartheta\left(P_{1}\right)=P_{1}$, we have

$$
\int_{P_{1}}^{\vartheta(z)} \eta=-\int_{\vartheta\left(P_{1}\right)}^{\vartheta(z)} \vartheta^{*} \bar{\eta}=-\int_{P_{1}}^{z} \bar{\eta} .
$$

Thus

$$
\operatorname{Re} \int_{P_{1}}^{z} \eta+\operatorname{Re} \int_{P_{1}}^{\vartheta(z)} \eta=\operatorname{Re} \int_{P_{1}}^{z}(\eta-\bar{\eta})=0
$$

and (II.3) follows. Since $\vartheta(z)=z$ for $z \in \partial T, R(z)=1$, for $z \in \partial T$, to complete the proof.

Let us now introduce $\mathfrak{A}$ as the space of meromorphic functions on $S$ which are holomorphic on $S \backslash\left\{P_{0}, P_{\infty}\right\}$. We write

$$
\mathfrak{U}=\mathfrak{U}_{-} \oplus \mathfrak{U}_{1} \oplus \mathfrak{U}_{+},
$$

where $\mathfrak{U}_{-}$is the subspace of functions $f \in \mathfrak{U}$ with $\operatorname{ord}_{P_{0}} f \geqq 1$. Likewise $\mathfrak{U}_{+}$is the subspace of functions $f \in \mathfrak{U}$ with ord $\boldsymbol{P}_{\infty} f \geqq 1$. The space $\mathfrak{A}_{1}$ is $(g+1)$-dimensional and consists of functions $f \in \mathfrak{A}$ with ord $P_{0} f \leqq 0$ and $\operatorname{ord}_{P_{\infty}} f \leqq 0$.

The space $\mathfrak{U}$, or more precisely, the space $\mathfrak{A} /[1]$, carries a natural symplectic form

$$
(f, g):=\operatorname{Res}_{P_{0}}(d f \cdot g) .
$$

Note that

$$
(f, g)=-\operatorname{Res}_{P_{\infty}}(d f \cdot g)=\frac{1}{2 \pi i} \int_{C} d f \cdot g,
$$


where $C$ is any cycle separating $P_{0}$ from $P_{\infty}$. This form vanishes on $\mathfrak{A}_{+} \times \mathfrak{A}_{+}$and on $\mathfrak{U}_{-} \times \mathfrak{U}_{-}$.

We define an antilinear involution $\Theta: \mathfrak{A} \rightarrow \mathfrak{A}$ by

Clearly

$$
(\Theta f)(z):=\overline{f(\vartheta(z))} \text {. }
$$

$$
\Theta: \mathfrak{U}_{ \pm} \rightarrow \mathfrak{U}_{\mp}
$$

and

$$
\Theta: \mathfrak{U}_{1} \rightarrow \mathfrak{U}_{1} .
$$

The map $\Theta$ provides the Osterwalder-Schrader reflection on a Schottky double. In fact, we establish below that $\Theta$ gives rise to a natural inner product on $\mathfrak{A}_{+}$. We define

$$
\langle f, g\rangle:=(\Theta f, g) .
$$

Theorem II.2. The form (II.11) defines an inner product on $\mathfrak{U}_{+}$.

Proof. We verify that (II.11) is positive on $\mathfrak{U}_{+}$. We compute for $f \in \mathfrak{U}_{+}$,

$$
\operatorname{Res}_{P_{0}}(\Theta f, f)=\frac{1}{2 \pi i} \int_{\partial T} d \overline{(f(\vartheta(z)))} f(z)=-\frac{1}{2 \pi i} \int_{\partial T} \overline{f(z)} d f(z) .
$$

By Stokes' theorem, and the fact that $f$ is holomorphic on $\bar{T}$,

$$
\langle f, f\rangle=\frac{1}{2 \pi i} \int_{T} \overline{d f(z)} \wedge d f(z)=\frac{1}{\pi}\|d f\|_{L_{2}(T)}^{2} \geqq 0 .
$$

If $\langle f, f\rangle=0$, then $f=$ const; but $\operatorname{ord}_{P_{\infty}} f>0$, so $f=0$.

\section{The Heisenberg Algebra}

The space $\mathfrak{A}$ can be given the structure of an infinite-dimensional Lie algebra with the bracket

$$
[f, g]=(f, g) 1 \text {. }
$$

We call this algebra the Heisenberg algebra on $S$ and denote it, by abuse of notation, $\mathfrak{A}$. We study representations of $\mathfrak{A}$.

We can also interpret $\mathfrak{A}$ in terms of the Krichever-Novikov basis $\left\{f_{n}^{(0)}\right\}[\mathrm{KN}$, KL]. The function $f_{0}^{(0)}=1$, while $\mathfrak{A}_{-}$is spanned by the $f_{n}^{(0)}$ 's with $n \geqq 1$. The space $\mathfrak{U}_{+}$is spanned by $f_{n}^{(0)}$ with $n \leqq-(g+1)$. The $(g+1)$-dimensional space $\mathfrak{U}_{1}$ is spanned by $f_{n}^{(0)}$ 's with $-g \leqq n \leqq 0$. We can interpret $\mathfrak{A}_{+}$as creation operators and $\mathfrak{U}_{-}$as annihilation operators. The interpretation of $\mathfrak{U}_{1}$ is subtler and will be studied in the next two sections.

\section{The Extended Heisenberg Algebra}

In studying representations of $\mathfrak{A}$ we encounter the problem that the form $\langle\cdot, \cdot\rangle$ does not have a definite sign on $\mathfrak{U}_{1}$. To overcome this difficulty, we extend $\mathfrak{A}$ to a larger Lie algebra $\Omega$ for which the positivity problem has an easy solution. This is the extended Heisenberg algebra. 
In Sect. III we defined the Heisenberg algebra $\mathfrak{A}$ as the space of meromorphic functions on $S$ which are holomorphic on $S \backslash\left\{P_{0}, P_{\infty}\right\}$, equipped with the Lie bracket (III.1). Here we define the extended Heisenberg algebra $\Omega$ and an embedding of $\mathfrak{A}$ into $\Omega$. The algebra $\Omega$ is a space of meromorphic 1-forms on $S$ with a Lie bracket

$$
[\omega, \eta]=(\omega, \eta)_{*} \tau .
$$

Here $(\cdot, \cdot)_{*}$ is given in (IV.12) and $\tau$ is a central element of $\Omega$. We define an embedding $i: \mathfrak{A} \rightarrow \Omega$ and extend the construction of a reflection positive inner product to $\Omega$.

Let the Schottky double $S$ of $T$ have $\partial T$ consisting of $n$ connected components $\Gamma_{1}, \ldots, \Gamma_{n}$. With $g_{0}=$ genus $T$, we observe that genus $S=2 g_{0}+(n-1)$. We choose a symmetric homology basis for $S$ in a standard way, see $[\mathrm{F}]$. The basis $\left\{a_{1}, \ldots, a_{g}\right.$, $\left.b_{1}, \ldots, b_{g}\right\}$ has $2 g_{0}$ generators in $T, 2 g_{0}$ generators in the reflection $\bar{T}=\vartheta T$, and $2(n-1)$ generators which link $T$ and $\bar{T}$. Specifically, let

$$
\left\{a_{1}, \ldots, a_{g_{0}}, b_{1}, \ldots, b_{g_{0}}\right\} \subset T,
$$

and choose

$$
a_{i}=\Gamma_{i-g_{0}}, \quad b_{i}=-\vartheta b_{i}, \text { for } i=g_{0}+1, \ldots, g_{0}+(n-1) .
$$

Furthermore, let

$$
a_{g_{0}+(n-1)+i}=9 a_{i}, \quad b_{g_{0}+(n-1)+i}=-\vartheta b_{i},
$$

for $i=1,2, \ldots, g_{0}$.

Now we choose $\Omega$ as the space of meromorphic one-forms $\omega$ on $S$ such that and

(i) $\omega$ is holomorphic on $S \backslash\left\{P_{0}, P_{\infty}\right\}$,

(ii) $\int_{a_{j}} \omega=0, \quad j=1,2, \ldots, g$.

Furthermore, let $\tau \in \Omega$ be the unique one-form with first order poles and such that

$$
\operatorname{Res}_{P_{0}} \tau=i \text {. }
$$

We can now write

$$
\Omega=\Omega_{-} \oplus \Omega_{1} \oplus \Omega_{+},
$$

and for $\omega \in \Omega$ there is a unique decomposition

$$
\omega=\omega_{-}+\lambda \tau+\omega_{+} .
$$

Here $\Omega_{1}$ is spanned by $\tau, \lambda \in C$, and

$$
\begin{aligned}
& \operatorname{ord}_{P_{0}} \omega_{-} \geqq 0, \text { for } \omega_{-} \in \Omega_{-} \text {, } \\
& \operatorname{ord}_{P_{\infty}} \omega_{+} \geqq 0, \text { for } \omega_{+} \in \Omega_{+} \text {. }
\end{aligned}
$$

It follows that

$$
\operatorname{Res}_{P_{0}} \omega_{ \pm}=0
$$


and that

$$
\int_{a_{j}} \omega_{ \pm}=0, \quad j=1,2, \ldots, g .
$$

In this way we obtain for $\Omega$ a decomposition of the form (II.5), but with $\Omega_{1}$ onedimensional. Let

$$
\Omega=\Omega_{1} \oplus \Omega^{\prime}, \quad \Omega^{\prime}=\Omega_{+} \oplus \Omega_{-} .
$$

We define a quadratic form on $\Omega^{\prime} \times \Omega^{\prime}$ by

$$
(\omega, \eta)_{*}=\frac{1}{2 \pi i} \int_{\partial T} \omega \int_{P_{1}}^{z} \eta
$$

Theorem IV.1. The form (IV.12) is symplectic on $\Omega^{\prime} \times \Omega^{\prime}$. Furthermore, it vanishes on $\Omega_{+} \times \Omega_{+}$and $\Omega_{-} \times \Omega_{-}$.

Proof. Note that (IV.12) is well-defined, since the integral of $\eta$ around a cycle is a constant. Thus, even though $\int_{P_{1}}^{z} \eta$ is multivalued, as a consequence of (IV.9), $(\omega, \eta)_{*}$ is unaffected. We choose the path from $P_{1}$ to $z$ to lie in $T$ and not to intersect any cycle $a_{i}$ or $b_{i}, i=1,2, \ldots, g_{0}$.

Let us now verify that $(\omega, \eta)_{*}=-(\eta, \omega)_{*}$. Because of our choice above, we can represent the integral in (IV.12) as follows: Let $\widetilde{T}$ be a representation of $T$ as shown in Fig. 2 , obtained by cutting $T$ along the $2 g_{0}$ cycles $a_{1}, b_{1}, \ldots, a_{g_{0}}, b_{g_{0}}$. Hence $\widetilde{T}$ is a polygon with $4 g_{0}$ sides and $n$ holes, these holes bounded by $\Gamma_{1}, \ldots, \Gamma_{n}$.

Although $\widetilde{T}$ is not simply connected, the functions

$$
\int_{P_{1}}^{z} \omega \text { and } \int_{P_{1}}^{z} \eta
$$

are single-valued, meromorphic functions on $\widetilde{T}$. Here we use (IV.9) and $\int_{\Gamma_{2}} \omega=0$, for $\omega \in \Omega^{\prime}$, namely assumption (ii) for forms in $\Omega$. It follows that the product $\int_{P_{1}}^{z} \omega \int_{P_{1}}^{z} \eta$
has no logarithmic singularity and therefore

$$
\operatorname{Res}_{P_{0}} d\left(\int_{P_{1}}^{z} \omega \int_{P_{1}}^{z} \eta\right)=0 \text {. }
$$

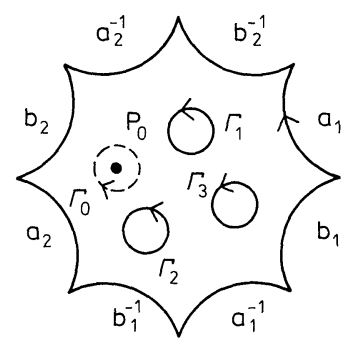

Fig. 2. An example of $\tilde{T}$ when $g_{0}=2$ and $n=3$. Here $\partial \tilde{T}$ is the set $\left(a_{1}, b_{1}, a_{1}^{-1}, b_{1}^{-1}, a_{2}, b_{2}, a_{2}^{-1}, b_{2}^{-1}\right.$, $\left.\Gamma_{1}, \Gamma_{2}, \Gamma_{3}\right)$ 
Let us compute

$$
(\omega, \eta)_{*}+(\eta, \omega)_{*}=\frac{1}{2 \pi i} \int_{\partial T}\left(\omega \int_{P_{1}}^{z} \eta+\eta \int_{P_{1}}^{z} \omega\right) .
$$

Introduce a cycle $\Gamma_{0}$ about $P_{0}$, and let

$$
\dot{T}=\tilde{T} \backslash \operatorname{Int} \Gamma_{0}, \quad \text { with } \quad \partial \dot{T}=\partial \widetilde{T} \cup \Gamma_{0} .
$$

By Stokes' theorem we can write

$$
\begin{aligned}
(\omega, \eta)_{*}+(\eta, \omega)_{*}= & \frac{1}{2 \pi i} \int_{T} d\left(\omega \int_{P_{1}}^{z} \eta+\eta \int_{P_{1}}^{z} \omega\right) \\
& -\frac{1}{2 \pi i} \int_{\partial T \backslash \partial T}\left(\omega \int_{P_{1}}^{z} \eta+\eta \int_{P_{1}}^{z} \omega\right) .
\end{aligned}
$$

Clearly, the first term on the right-hand side of (IV.16) vanishes. We claim that the second term also vanishes. To verify this, let us write

$$
\omega \int_{P_{1}}^{z} \eta=\omega f
$$

where $f$ is a holomorphic function on $\dot{T}$. Then

$$
\underset{\partial T \backslash \partial T}{ } \omega f=\int_{\Gamma_{0}} \omega f+\sum_{i=1}^{g_{0}}\left(\int_{a_{i}} \omega f+\int_{b_{i}} \omega f+\int_{a_{i}^{-1}} \omega f+\int_{b_{i}^{-1}} \omega f\right) .
$$

Observe that

$$
\int_{a_{i}^{-1}} \omega f=-\int_{a_{i}} \omega\left(f+\int_{b_{i}} \eta\right)
$$

Thus the integrals over $a_{i}$ and $a_{i}^{-1}$ cancel, as $\int_{a_{i}} \omega=0$. Likewise, the integrals over $b_{i}$ and $b_{i}^{-1}$ cancel. Hence

$$
\int_{\partial T} \omega f=-2 \pi i \operatorname{Res}_{P_{0}}(\omega f) .
$$

Similarly we obtain

$$
\begin{aligned}
-\frac{1}{2 \pi i} \int_{\partial \dot{T} \backslash \partial T}\left(\omega \int_{P_{1}}^{z} \eta+\eta \int_{P_{1}}^{z} \omega\right) & =\operatorname{Res}_{P_{0}}\left(\omega \int_{P_{1}}^{z} \eta+\eta \int_{P_{1}}^{z} \omega\right) \\
& =\operatorname{Res}_{P_{0}} d\left(\int_{P_{1}}^{z} \omega \int_{P_{1}}^{z} \eta\right)=0
\end{aligned}
$$

where we used (IV.13) to conclude that (IV.18) is zero. Thus we have verified that

$$
(\omega, \eta)_{*}+(\eta, \omega)_{*}=0
$$

Furthermore, as a consequence of Theorem IV.2 below, we find that $(\cdot, \cdot)_{*}$ is nondegenerate. We conclude that $(\cdot, \cdot)_{*}$ is symplectic. 
We now check that $(\cdot, \cdot)_{*}$ vanishes on $\Omega_{-} \times \Omega_{-}$. We repeat the above computation to obtain

$$
(\omega, \eta)_{*}=\operatorname{Res}_{P_{0}}\left(\omega \int_{P_{1}}^{z} \eta\right)
$$

If $\omega, \eta \in \Omega_{-}$, they are holomorphic at $P_{0}$ so the residue vanishes. Similarly, for $\omega$, $\eta \in \Omega_{+}$we obtain a representation on the reflection of $\dot{T}$, and we conclude that $(\omega, \eta)_{*}$ also vanishes. This completes the proof of the theorem.

We note that the form $(\cdot, \cdot)_{*}$ has not been defined on $\Omega \times \Omega$. We extend the definition to this case by setting

$$
(\tau, \omega)_{*}:=0, \quad \omega \in \Omega .
$$

Let us also define an antilinear involution $\Theta$ on $\Omega$ by

$$
\Theta \omega:=\overline{\vartheta^{*} \omega}
$$

where $\vartheta^{*}$ is the lift of $\vartheta$ from $S$ to 1 -forms. With this definition

$$
\Theta: \Omega_{ \pm} \rightarrow \Omega_{\mp} \quad \text { and } \quad \Theta \tau=\tau .
$$

We now define a sesquilinear form $\langle\cdot, \cdot\rangle_{*}$ on $\Omega \times \Omega$ by

$$
\langle\omega, \eta\rangle_{*}:=(\Theta \omega, \eta)_{*} .
$$

Theorem IV.2. The form $\langle\cdot, \cdot\rangle_{*}$ defines a Hermitian inner product on $\Omega_{+}$.

Proof. We compute as in the proof of Theorem II.1. Then

$$
\langle\omega, \omega\rangle_{*}=(\Theta \omega, \omega)_{*}=-\frac{1}{2 \pi i} \int_{\partial T} \omega \int_{P_{1}}^{z} \overline{\vartheta^{*} \omega}=-\frac{1}{2 \pi i} \int_{\partial T} \omega \int_{P_{1}}^{z} \bar{\omega}
$$

using the invariance of $\partial T$ under $\vartheta$. Cutting $\vartheta T$ as explained in the proof of Theorem IV.1 and using Stokes' theorem we find

$$
\langle\omega, \omega\rangle_{*}=\frac{1}{2 \pi i} \int_{\partial \bar{T}} \omega \int_{P_{1}}^{z} \bar{\omega}=\frac{1}{2 \pi i} \int_{\bar{T}} d\left(\bar{\omega} \int_{P_{1}}^{z} \omega\right)=\frac{1}{2 \pi i} \int_{\bar{T}} \bar{\omega} \wedge \omega .
$$

Other terms arising from the boundary of $\widetilde{\vartheta T}$ do not contribute, as in the proof of Theorem IV.1. The positivity of (IV.26) follows as does the positivity of (II.12), namely

$$
\langle\omega, \omega\rangle_{*}=\frac{1}{\pi}\|\omega\|_{L_{2}(\widetilde{T})}^{2} \geqq 0
$$

Furthermore, it is clear that $\langle\omega, \omega\rangle_{*}=0$, if and only if $\omega \equiv 0$.

We now consider the embedding

$$
i: \mathfrak{U} \rightarrow \Omega .
$$

Let $f \in \mathfrak{U}_{ \pm}$. We define

$$
i(f):=d f \in \Omega_{ \pm}
$$


In fact, since $f$ is single-valued and meromorphic, the integral of $d f$ on a cycle vanishes. We choose a point $P \neq P_{0}, P_{\infty}, P \in \partial T$ and set for $f \in \mathfrak{U}_{1}$,

$$
i(f):=d f+f(P) \tau \text {. }
$$

Theorem IV.3. With these definitions, $i$ is an injective homomorphism of Lie algebras. In particular,

$$
(f, g)=(i(f), i(g))_{*}
$$

Moreover,

$$
\Theta i=i \Theta
$$

Proof. We use the representation (IV.20) for $f, g \in \mathfrak{U}_{ \pm}$. Then

$$
(i(f), i(g))_{*}=\operatorname{Res}_{P_{0}}\left(d f \int_{P_{1}}^{z} d g\right)=\operatorname{Res}_{P_{0}}(d f \cdot g),
$$

since as $f$ is meromorphic, $d f$ has no residue at $P_{0}$. But this agrees with definition (II.6). For $f \in \mathfrak{U}_{1}$ or $g \in \mathfrak{U}_{1}$, we must add $f(P) \tau$ or $g(P) \tau$ to $i(f)$ or $i(g)$. But this does not contribute to $(i(f), i(g))_{*}$ so (IV.31) holds in all cases. The remaining statements are clear.

Proposition IV.4. The space $\Omega / i(\mathfrak{U})$ is g-dimensional.

Proof. Let $\Lambda$ be the space of meromorphic 1-forms $\xi$ on $S$ such that $\operatorname{ord}_{P} \xi \geqq 0$, for $P$ $\neq P_{\infty}$, and $\operatorname{ord}_{P_{\infty}} \xi \geqq-(g+1)$. It follows immediately from the Riemann-Roch theorem that $\operatorname{dim} \Lambda=2 g$. Now, for any meromorphic 1 -form $\omega$ which is holomorphic on $S \backslash\left\{P_{0}, P_{\infty}\right\}$, there is a function $f \in \mathfrak{A}$ such that $\omega-i(f) \in \Lambda$. Therefore, $\operatorname{dim}(\Omega \cap \Lambda)=2 g-g=g$, as claimed.

We remark that the functional $\lambda$ defined on $\mathscr{A}_{1}$ by $\lambda(f)=f(P)$ satisfies

$$
\lambda(\Theta f)=\overline{\lambda(f)} .
$$

This functional is a parameter of our embedding of $\mathfrak{A}$ into $\Omega$.

\section{Representations}

We construct representations $\Phi$ of the algebra $\mathfrak{A}$ by unbounded operators on a Hilbert space $\mathscr{H}$. We require

(i) $\Phi$ is a homomorphism of $\mathfrak{U}$ into a Lie algebra of unbounded operators on $\mathscr{H}$, with $\Phi(1)=I$.

(ii) $\mathscr{D}$ is a common, dense, invariant domain for the operators $\Phi(f), f \in \mathfrak{A}$.

(iii) The $\Phi$ satisfy $\Phi(f)^{*}=\Phi(\Theta f)$ on $\mathscr{D}$.

(iv) $\mathscr{D}$ contains a unique vector $v$ which is cyclic for $\Phi(\mathfrak{A})$, and

$$
\Phi\left(\mathfrak{A}_{-}\right) v=0 \text {. }
$$

We first construct a corresponding representation for the extended algebra $\Omega$. In that case we have:

$\left(\mathrm{i}^{\sim}\right) \widetilde{\Phi}$ is a homomorphism of $\Omega$ into an algebra of operators on $\mathscr{H}$ with $\widetilde{\Phi}(\tau)=I$. 
(ii $\sim$ )-(iv $\sim$ ) as above, with $\Omega$ replacing $\mathfrak{A}$.

The conditions $\left(\mathrm{i}-\mathrm{i} \mathrm{v}^{\sim}\right)$ characterize a representation uniquely, and the representation can be constructed as follows. Let $S\left(\Omega_{+}\right)$be the symmetric tensor algebra over $\Omega_{+}$. That is

$$
S\left(\Omega_{+}\right)=\bigoplus_{n \geqq 0} \bigotimes_{s}^{n} \Omega_{+} .
$$

Let $\mathscr{H}$ be the Hilbert space obtained by completion of $S\left(\Omega_{+}\right)$in the norm defined by $\langle\cdot, \cdot\rangle_{*}$ of (IV.24). Let $k: S\left(\Omega_{+}\right) \rightarrow \mathscr{H}$ be the natural embedding, and define $\mathscr{D}=k\left(S\left(\Omega_{+}\right)\right)$. Let

$$
v=k((1,0,0, \ldots))
$$

For $\omega_{1}, \ldots, \omega_{n} \in \Omega_{+}$, define

$$
v_{n}\left(\omega_{1}, \ldots, \omega_{n}\right)=k\left(\left(0,0, \ldots, \omega_{1} \otimes_{s} \ldots \otimes_{s} \omega_{n}, 0, \ldots\right)\right) .
$$

These vectors span $\mathscr{H}$ and there is a Fock type construction

$\tilde{\Phi}(\omega) v_{n}\left(\omega_{1}, \ldots, \omega_{n}\right)=\left\{\begin{array}{cl}v_{n+1}\left(\omega, \omega_{1}, \ldots, \omega_{n}\right), & \omega \in \Omega_{+}, \\ \sum_{j=1}^{n}\left(\omega, \omega_{j}\right)_{*} v_{n-1}\left(\omega_{1}, \ldots, \hat{\omega}_{j}, \ldots, \omega_{n}\right), & \omega \in \Omega_{-},\end{array}\right.$

where $\hat{\omega}$ denotes the omission of $\omega$. Also, we set $\widetilde{\Phi}(\tau)=I$. Then $\left(\mathrm{i}^{\sim}-\mathrm{i} \mathrm{v}^{\sim}\right)$ follow by standard arguments.

We construct a representation $\Phi$ of $\mathfrak{A}$ from the representation $\widetilde{\Phi}$ of $\Omega$ as follows: for $f \in \mathfrak{A}$, let

$$
\Phi(f)=\widetilde{\Phi}(i(f)) .
$$

Thus for $f \in \mathfrak{A}_{ \pm}, \Phi(f)=\widetilde{\Phi}(d f)$. If $f \in \mathfrak{A}_{1}$, then

$$
i(f)=a_{f}+c_{f}+\lambda(f) \tau,
$$

where $a_{f} \in \Omega_{-}, c_{f} \in \Omega_{+}$and

$$
\Phi(f)=\widetilde{\Phi}\left(a_{f}\right)+\widetilde{\Phi}\left(c_{f}\right)+\lambda(f) I .
$$

Theorem V.1. The quadruple $(\mathscr{H}, \mathscr{D}, \Phi, v)$ is a representation of $\mathfrak{A}$ on the Hilbert space $\mathscr{H}$ which satisfies (i-iv).

Proof. We verify only that

$$
\Phi(f)^{*}=\Phi(\Theta f), \quad f \in \mathfrak{A},
$$

and

$$
[\Phi(f), \Phi(g)]=(f, g), \quad f, g \in \mathfrak{A},
$$

leaving the rest of the proof to the reader.

From (IV.32) $\Theta i(f)=i(\Theta f)$, and thus

$$
\Phi(f)^{*}=\widetilde{\Phi}(i(f))^{*}=\widetilde{\Phi}(\Theta i(f))=\widetilde{\Phi}(i(\Theta f))=\Phi(\Theta f),
$$

which is (V.9). 
To prove (V.10) we use (IV.31):

$$
[\Phi(f), \Phi(g)]=[\widetilde{\Phi}(i(f)), \tilde{\Phi}(i(g))]=(i(f), i(g))_{*}=(f, g),
$$

as claimed.

Remark. Note that as a consequence of (IV.32) and the uniqueness of the decomposition (V.7),

$$
\Theta a_{f}=c_{\Theta f} .
$$

Note also that the vacuum expectation value of $\Phi(f)$ is nonzero, if $f \in \mathfrak{A}_{1}$. In fact,

$$
\langle v, \Phi(f) v\rangle_{\mathscr{H}}=\lambda(f) .
$$

\section{References}

[A] Abikoff, W.: The real analytic theory of Teichmüller spaces. Lecture Notes in Mathematics, vol. 820. Berlin, Heidelberg, New York: Springer 1980

[BKS] Budzynski, R., Klimek, S., Sadowski, P.: The group of local biholomorphisms of $\mathbb{C}^{1}$ and conformal field theory in the operator formalism. Commun. Math. Phys. 121, 481-499 (1989)

[DVV] Dijkgraaf, R., Verlinde, E., Verlinde, H.: $c=1$ Conformal field theories on Riemann surfaces. Commun. Math. Phys. 115, 649-690 (1988)

[F] Fay, J.D.: Theta functions on Riemann surfaces. Berlin, Heidelberg, New York: Springer 1973

[FK] Farkas, H.M., Kra, I.: Riemann surfaces. Berlin, Heidelberg, New York: Springer 1980

[KL] Klimek, S., Lesniewski, A.: Global Laurent expansions on Riemann surfaces. Commun. Math. Phys. 125, 597-611 (1989)

[KN] Krichever, I.M., Novikov, S.P.: Algebras of Virasoro type, Riemann surfaces and structures of the theory of solutions. Funct. Anal. Pril. 21, 126-142 (1987) and Virasoro type algebras, Riemann surfaces and strings in Minkowski space. ibid, 294-307

Communicated by A. Jaffe

Received April 21, 1989 
